\title{
Avaliação do efeito hipoglicemiante de Bauhinia forficata (pata-de-vaca) e sua aplicabilidade no tratamento de diabetes mellitus tipo II
}

\author{
Evaluation of the hypoglycemiating effect of Bauhinia forficata (pata-de-vaca) and its \\ applicability in the treatment of diabetes mellitus type II \\ Evaluación del efecto hipoglucemiante de Bauhinia forficata (pata-de-vaca) y su \\ aplicabilidad en el tratamiento de la diabetes mellitus tipo II
}

Vanessa Gonçalves de Moraes ${ }^{1}$, Wendy Clésia Maciel dos Santos ${ }^{1}$, lasmin Leticia dos Santos Monteiro $^{2}$, Antônio Rafael Quadros Gomes ${ }^{1,2}$, Sabrina de Carvalho Cartágenes ${ }^{1,2}$, Heliton Patrick Cordovil Brígido ${ }^{1,2, *}$.

\section{RESUMO}

Objetivo: Realizar uma revisão bibliográfica acerca dos efeitos hipoglicemiantes de Bauhinia forficata (patade-vaca) e sua aplicabilidade no tratamento de diabetes mellitus tipo II. Métodos: Trata-se de um estudo transversal, exploratório, qualitativo e descritivo, a partir das bases de dados científicas: Medline via PubMed, Science Direct e Google Scholar, por meio dos descritores "Bauhinia forficata" e "Diabetes", no recorte temporal de 2017 a 2021. Foi realizada a leitura dos títulos e resumos dos artigos encontrados e selecionados nove $(n=9)$ artigos que continham procedimentos experimentais de avaliação da ação hipoglicemiante patade-vaca e aplicação no tratamento de diabetes tipo II. Resultados: As evidências científicas comprovam a presença de flavonóides responsáveis pela atividade hipoglicemiante da Bauhinia forficata, resultando na redução dos níveis séricos de glicose, em diversas concentrações da planta medicinal e formas farmacêuticas. Considerações finais: A Bauhinia forficata apresenta ação benéfica sobre a redução de glicose no organismo, apresenta segurança no uso, é acessível e economicamente viável, podendo ser utilizada como tratamento complementar de diabetes mellitus tipo II, desde que seu uso seja racional e orientado por profissionais de saúde.

Palavras-chave: Plantas medicinais, Diabetes Mellitus, Hipoglicemiante, Etnofarmacologia.

\section{ABSTRACT}

Objective: To carry out a literature review about the hypoglycemic effects of Bauhinia forficata (pata-de-vaca) and its applicability in the treatment of type II diabetes mellitus. Methods: This is a cross-sectional, exploratory, qualitative and descriptive study, based on the scientific databases: Medline via PubMed, Science Direct and Google Scholar, using the descriptors "Bauhinia forficata" and "Diabetes", in the time frame. from 2017 to 2021. The titles and abstracts of the articles found were read and nine $(n=9)$ articles were selected that contained experimental procedures for evaluating the hypoglycemic action of cow's foot and application in the treatment of type II diabetes. Results: Scientific evidence proves the presence of flavonoids responsible for the hypoglycemic activity of Bauhinia forficata, resulting in the reduction of serum glucose levels, in different concentrations of the medicinal plant and pharmaceutical forms. Final considerations: That Bauhinia forficata has a beneficial action on the reduction of glucose in the body, is safe in use, is accessible and economically viable, and can be used as a complementary treatment for type II diabetes mellitus, provided that its use is rational. and guided by health professionals.

Keywords: Medicinal plants, Diabetes Mellitus, Hypoglycemic, Ethnopharmacology.

\section{RESUMÉN}

Objetivo: Realizar una revisión bibliográfica sobre los efectos hipoglucemiantes de Bauhinia forficata (patade-vaca) y su aplicabilidad en el tratamiento de la diabetes mellitus tipo II. Métodos: Se trata de un estudio transversal, exploratorio, cualitativo y descriptivo, basado en las bases de datos científicas: Medline vía

1 Faculdade Cosmopolita, Belém - PA. *E-mail: helitombrigido@gmail.com

2 Universidade Federal do Pará, Belém - PA. 
PubMed, Science Direct y Google Scholar, utilizando los descriptores "Bauhinia forficata" y "Diabetes", en el marco temporal de 2017 a 2021. Se leyeron los títulos y resúmenes de los artículos encontrados y se seleccionaron nueve $(n=9)$ artículos que contenían procedimientos experimentales para evaluar la acción hipoglucemiante de la pata de vaca y su aplicación en el tratamiento de la diabetes tipo II. Resultados: La evidencia científica comprueba la presencia de flavonoides responsables de la actividad hipoglucemiante de Bauhinia forficata, resultando en la reducción de los niveles de glucosa sérica, en diferentes concentraciones de la planta medicinal y formas farmacéuticas. Consideraciones finales: La Bauhinia forficata tiene una acción benéfica en la reducción de la glucosa en el organismo, es segura en su uso, accesible y económicamente viable, y puede ser utilizada como tratamiento complementario para la diabetes mellitus tipo II, siempre que su el uso es racional y guiado por profesionales de la salud.

Palabras clave: Plantas medicinales, Diabetes Mellitus, Hipoglucemiantes, Etnofarmacología.

\section{INTRODUÇÃO}

Diabetes mellitus (DM) é uma doença crônica, multifatorial, caracterizada por altos níveis de glicose circulantes na corrente sanguínea. Esta patologia representa um grave problema de saúde pública, uma vez que, cerca de 463 milhões de pessoas possuem diabetes em todo o mundo, dentre estes, 13 milhões de brasileiros. Os gastos públicos com o diabetes mellitus giram em torno de US $\$ 760$ bilhões ao ano e esta doença contribui em até 50\% para cardiopatias, acidente vascular cerebral, hipertensão arterial e síndrome metabólica (NASCIMENTO EMM, et al., 2021).

Diabetes mellitus, segundo classificação da Associação Americana de Diabetes (ADA) é dividida de acordo com aspectos fisiopatológicos, principalmente em tipo 1 e tipo 2: o diabetes tipo 1 é caracterizado pela destruição de Células $\beta$ pancreáticas e deficiência absoluta de insulina, já o diabetes Tipo 2 ocorre devido a resistência à insulina ou deficiência parcial de insulina (CARVALHO AC, et al., 2021).

De acordo com a Sociedade Brasileira de Diabetes (SBD), os valores de normalidade da glicose no organismo em jejum devem ser inferiores a 99mg/dl. Um indivíduo entre 99 a $110 \mathrm{mg} / \mathrm{dl}$ são considerados pré-diabéticos. Acima de $110 \mathrm{mg} / \mathrm{dl}$ são indivíduos considerados diabéticos. A educação alimentar, a prática de atividades físicas regulares e o controle dos níveis de glicemia capilar são imprescindíveis para o acompanhamento e controle da doença, uma vez que, acompanhar os índices pode determinar ações em saúde capazes de evitar as complicações decorrentes do diabetes mellitus (SBD, 2000).

Os agravos da Diabetes Mellitus são inúmeros, dentre eles, a cegueira, amputações de membros inferiores, insuficiência renal, hemodiálise, pé diabético, úlceras cutâneas, neuropatias, macrovasculopatia, susceptibilidade a infecção, deformidades cutâneas, lesões hepáticas, agravamento de lesões cardíacas e síndrome metabólica. Os principais fatores de risco para o desenvolvimento do DM são: hipertensão arterial sistêmica, hipercolesterolemia, obesidade, Índice de Massa Corporal (IMC) elevado, alta circunferência da cintura, fatores hereditários, doenças renais crônicas, síndrome de ovários policísticos, sedentarismo, tabagismo, alto consumo de sal e açúcares, alimentação inadequada, distúrbios psiquiátricos, desregulação hormonal e do sono, além do uso de glicocorticoides (SILVA AD, et al., 2020; FONSECA KP e RACHED CDA, 2019).

O tratamento é estruturado de forma diferente de acordo com a classificação do diabetes Mellitus. $O$ paciente portador de diabetes tipo 1, não possui nenhuma capacidade de produzir insulina, portanto, depende do uso externo para controle dos níveis glicêmicos. Para o portador de diabetes tipo 2, a recomendação decorre da associação de medidas farmacológicas, representadas pelos medicamentos e/ou insulina e medidas não farmacológicas, que podem ser práticas regular de atividade física, controle da alimentação, ingestão de água, redução do Índice de Massa Corporal (IMC) e circunferência abdominal, entre outras recomendações, visando a prevenção de complicações (SANTOS AL, et al., 2020).

O tratamento com ativos hipoglicemiantes é o mais comum, no entanto, objetivando a qualidade de vida e redução dos eventos adversos a medicamentos, surgem neste contexto, as plantas medicinais. As plantas medicinais são espécimes vegetais capazes de produzir princípios ativos que apresentem ação terapêutica (FIGUEIREDO LB e PAIVA PMH, 2020). 
A relevância deste artigo se dá pelo fato da Bauhinia forficata (pata-de-vaca) ser utilizada na região amazônica há muitos anos como hipoglicemiante e pode ser utilizada como tratamento complementar no diabetes mellitus, oportunizando a grande disponibilidade de flora brasileira e baixo custo, com grandes benefícios a seus usuários. Diante do exposto, este artigo objetivou realizar uma revisão bibliográfica acerca dos efeitos hipoglicemiantes de Bauhinia forficata (pata-de-vaca) e sua aplicabilidade no tratamento de diabetes mellitus tipo II.

\section{MÉTODOS}

Trata-se de uma revisão bibliográfica, de caráter transversal, qualitativo, exploratório e descritivo, realizada por meio da pesquisa nas bases de dados científicas: Medline via PubMed, Science Direct e Google Scholar. Os descritores utilizados foram: "Bauhinia forficata" e "Diabetes", com o auxílio do operador booleano AND, nos idiomas português e inglês e recorte temporal de 2017 a 2021. Para filtros específicos, foram utilizadas etapas sequenciadas descritas na Figura 1.

Após uma seleção criteriosa, foram encontrados, $(n=3)$ artigos na Medline/PubMed, $(n=3)$ na Science Direct e $(n=12)$ Google Scholar. Foram excluídos artigos repetidos nas bases, selecionando-se $(n=9)$ artigos que continham como temática a avaliação da ação hipoglicemiante da pata-de-vaca e seu uso para 0 tratamento de diabetes tipo II. Vale ressaltar que foram excluídos da pesquisa, monografias, teses e dissertações, avaliando somente artigos com procedimentos experimentais acerca da $B$. forficata. em seres humanos.

Figura 1 - Etapas sequenciais de seleção dos artigos componentes do estudo.

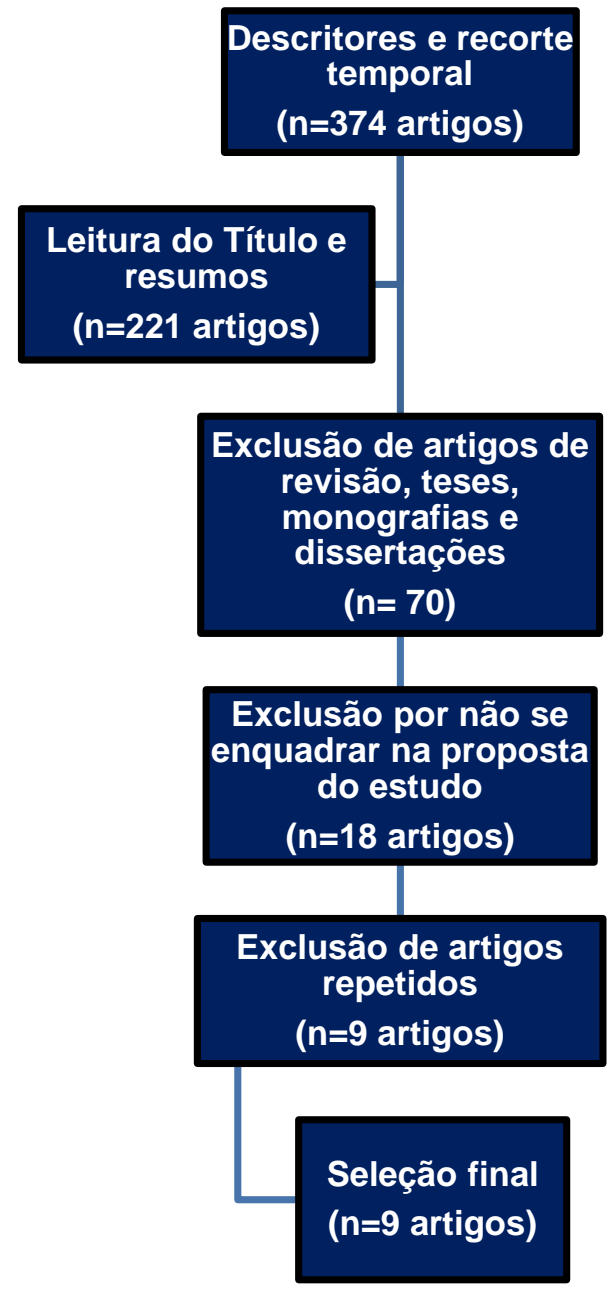

Fonte: Moraes VG, et al., 2022. 


\section{RESULTADOS E DISCUSSÃO}

A partir dos $(n=9)$ artigos selecionados, foi elaborado o Quadro 1 para melhor visualização dos artigos, ressaltando que todos os estudos são provenientes de procedimentos experimentais realizados com espécime vegetal Bauhinia forficata, avaliando sua ação hipoglicemiante.

Quadro 1 - Síntese dos artigos avaliados acerca da eficácia terapêutica da Bauhinia forficata (pata-de-vaca) no tratamento de diabetes mellitus.

\begin{tabular}{|c|c|c|}
\hline Autores/Ano & Metodologia & Principais resultados \\
\hline $\begin{array}{l}\text { FARIAS FL, et al. } \\
(2018)\end{array}$ & $\begin{array}{l}\text { Estudo analítico } \\
\text { experimental in vitro }\end{array}$ & $\begin{array}{l}\mathrm{O} \text { extrato bruto da folha apresentou ampla atividade } \\
\text { antibacteriana frente às bactérias de origem hospitalar. }\end{array}$ \\
\hline $\begin{array}{l}\text { SILVA HGN, et } \\
\text { al. (2018) }\end{array}$ & $\begin{array}{l}\text { Pesquisa quantitativa, } \\
\text { transversal, } \\
\text { observacional e } \\
\text { descritiva }\end{array}$ & $\begin{array}{l}\text { Dentre } 71 \text { pessoas cadastradas no programa HIPERDIA, } \\
66,19 \% \text { utilizavam plantas medicinais para o tratamento da } \\
\text { diabetes, sendo a infusão das folhas da } B \text {. forficata a mais } \\
\text { frequente, em } 38,29 \% \text { dos casos. }\end{array}$ \\
\hline $\begin{array}{l}\text { CECHINEL- } \\
\text { ZANCHETT CC, } \\
\text { et al. (2019) }\end{array}$ & Estudo experimental & $\begin{array}{l}\text { As folhas de pata-de-vaca possuem atividade antidiabética } \\
\text { comprovada e efeito vasorrelaxante, modulando o tônus } \\
\text { vascular, podendo ser utilizada para tratamento de } \\
\text { doenças cardiovasculares. }\end{array}$ \\
\hline $\begin{array}{l}\text { MARIÁNGEL PC, } \\
\text { et al. (2019) }\end{array}$ & Estudo experimental & $\begin{array}{l}\text { B. forficata pode ajudar a reduzir os níveis de alguns } \\
\text { parâmetros do perfil lipídico }\end{array}$ \\
\hline $\begin{array}{l}\text { PINAFO MS, et } \\
\text { al. (2019) }\end{array}$ & Estudo experimental & $\begin{array}{l}\text { O extrato de } B . \text { forficata possui propriedades } \\
\text { hipoglicêmicas e antioxidantes capazes de minimizar os } \\
\text { efeitos do Bisphenol A (BPA). }\end{array}$ \\
\hline $\begin{array}{l}\text { FRANCO RR, et } \\
\text { al. (2020) }\end{array}$ & Estudo experimental & $\begin{array}{l}\text { Fitomarcadores utilizados na Bauhinia forficata } \\
\text { evidenciaram que todas as frações apresentaram atividade } \\
\text { antioxidante e antiglicemiante, além da capacidade de } \\
\text { inibição de enzimas digestivas. }\end{array}$ \\
\hline $\begin{array}{l}\text { PRADO IO, et al. } \\
(2020)\end{array}$ & Estudo experimental & $\begin{array}{l}\text { O chá de Bauhinia forficata pode ser útil na redução dos } \\
\text { níveis de glicose. }\end{array}$ \\
\hline $\begin{array}{l}\text { JUNG EP, et al. } \\
\qquad(2021)\end{array}$ & Estudo experimental & $\begin{array}{l}\text { O extrato contém kaempferol e quercetina, indicando que } \\
\text { as folhas de Bauhinia forficata são uma fonte importante de } \\
\text { compostos antioxidantes e hipoglicemiantes. }\end{array}$ \\
\hline $\begin{array}{l}\text { TONELLI CA, et } \\
\text { al. (2021) }\end{array}$ & $\begin{array}{l}\text { Ensaio clínico duplo- } \\
\text { cego }\end{array}$ & $\begin{array}{l}\text { O grupo A apresentou redução dos níveis de glicose e } \\
\text { triglicerídeos, em relação ao grupo B (placebo). A pata-de- } \\
\text { vaca reduz os processos inflamatórios e níveis elevados de } \\
\text { glicose no sangue. }\end{array}$ \\
\hline
\end{tabular}

Fonte: Moraes VG, et al., 2022.

\section{Panorama e tratamento de diabetes mellitus}

Para Brito VP, et al. (2020), Diabetes Mellitus representa um grave problema de saúde pública no Brasil. O tratamento alopático para equilibrar as taxas de açúcar no sangue são: a insulina e medicamentos hipoglicemiantes orais. Tais produtos são efetivos, mas possuem custo elevado e apresentam efeitos colaterais principalmente relacionados ao trato gastrointestinal, tais como: anorexia, náuseas, diarreia e desconforto abdominal. Contudo, a busca por novas opções de tratamento, levam ao seguimento de inserção de plantas medicinais devido ao baixo custo, segurança e alta disponibilidade no país.

Para Figueiredo LB e Paiva PMH (2020), diante de uma patologia como o diabetes mellitus, de grandes complicações e agravos a saúde do paciente, faz-se necessário um tratamento medicamentoso efetivo, visando aumentar a expectativa e qualidade de vida do paciente, bem como reduzir os riscos de complicações associadas ao diabetes. São recomendações vitais, a alimentação correta e adequações de exercícios físicos, redução de estresse, circunferência abdominal e uso de medicamentos hipoglicemiantes. No entanto, estes medicamentos podem levar ao aumento de peso, hipoglicemia, edemas, problemas gastrointestinais e resistência à insulina. Neste ínterim, a pata-de-vaca conduz a benefícios de baixa da glicemia capilar, com segurança, eficácia e alta adesão pelo paciente. 
Como enfatiza Xavier AT e Nunes JS (2018), diante do dado da Sociedade Brasileira de diabetes (SBD), que afirma haver mais de 13 milhões de pessoas diagnosticadas com diabetes no Brasil, representando em média, 6,9\% da população, faz-se necessário a busca por alternativas terapêuticas capazes de auxiliar o tratamento da população em geral. Estes autores realizaram uma revisão sistemática nas bases de dados científicas, encontrando como espécie vegetal mais utilizada no tratamento de diabetes mellitus, a $B$. forficata, com inúmeros indicações profissionais e experimentos que comprovam sua eficácia no tratamento da patologia.

\section{Surgimento da utilização da pata de vaca}

Segundo Figueiredo LB e Paiva PMH (2020) e Cardoso RO, et al. (2019), as plantas medicinais são utilizadas por muitos anos, devido a difusão de conhecimento acerca destes espécimes vegetais por sociedades tradicionais, de uso popular, empírico e cultural. As plantas medicinais por vezes são a única forma de tratamento, possivelmente em casos de inacessibilidade ao tratamento medicamentoso convencional ou devido ao alto custo dos medicamentos. A ação terapêutica da Bauhinia forficata é decorrente da presença de metabólitos secundários, que desenvolvem ação de defesa e proteção da planta, tais como flavonoides, alcaloides, taninos, terpenos, entre outros.

Para Guimarães BM, et al. (2021), a cultura e o cultivo das plantas medicinais em comunidades interioranas do Brasil traduzem-se em saúde e sustentabilidade ambiental. Segundo os autores, o conhecimento popular repassado por gerações acerca das plantas medicinais foram fontes geradoras de curiosidade, alvo de pesquisa e produção científica. Quanto mais estudos sobre as plantas medicinais, maior os subsídios para pesquisas experimentais e produção de medicamentos, Guimarães BM, et al. (2021) ainda destaca que os estudos com plantas medicinais de potencial hipoglicemiante por meio de embasamento na sabedoria popular, permitiu a elaboração de medicamentos modernos usados no tratamento da Diabetes Mellitus, como a Metformina, derivada da Galega officinales

Segundo Souza ID, et al. (2020), A Organização mundial da Saúde (OMS) estima que 80\% da população mundial utiliza tratamento medicamentoso a partir de plantas medicinais. As sociedades tradicionais, incluindo as tribos indígenas, foram sendo estudadas ao longo dos anos e este processo levou a produção de inúmeros estudos de comprovação científica acerca da utilização de plantas medicinais no tratamento de inúmeras doenças. O aumento por esta alternativa complementar vem crescendo em decorrência da alta disponibilidade das plantas medicinais no país, baixo custo e reduzidos efeitos colaterais. Diante de doenças, como o diabetes mellitus, é necessário que se evite complicações associadas, e utilize as plantas medicinais como tratamento complementar, aliados a redução de fatores de risco, como sedentarismo, má alimentação e o alto consumo de alimentos açucarados e industrializados.

De acordo com Carvalho AC, et al. (2021), o uso de plantas medicinais é regularizado pelo Decreto n5.813, de 22 de junho de 2006, conhecido como Política Nacional de Plantas Medicinais e Fitoterápicos. A partir deste decreto, foi possível ofertar ações diversas para promoção, prevenção e recuperação da saúde por meio do uso seguro e racional das plantas medicinais. As plantas medicinais dispõem de ação terapêutica, baixo custo, efetividade e redução dos efeitos colaterais quando comparadas a medicamentos alopáticos hipoglicemiantes orais no tratamento do Diabetes Mellitus. Apesar de apresentarem amplos benefícios, também podem vir a oferecer riscos mínimos, mas existentes se utilizadas de forma irracional, podendo ocasionar toxicidade, reações adversas e interações medicamentosas (BRIGIDO HPC, et al., 2021).

Para Guimarães BM, et al. (2021), a utilização de plantas medicinais efetivou-se como importante recurso para tratamentos de doenças crônicas, como o diabetes mellitus. Profissionais de saúde podem orientar os pacientes quanto ao uso seguro e racional das plantas medicinais, aproveitando os recursos disponíveis na flora brasileira, sumariamente na região amazônica, campo de maior biodiversidade vegetal do mundo. $O$ Ministério da Saúde, diante do uso de sociedade tradicionais e ampla utilização pela sociedade, criou a Política Nacional de Práticas Integrativas e Complementares, além de criar a Política Nacional de Plantas Medicinais e Fitoterápicos (PNPMF), a fim de garantir o uso seguro e efetivo das plantas medicinais. Já no ano de 2008, foi publicada a Relação Nacional de Plantas Medicinais de Interesse do SUS (RENISUS) abrangendo 71 plantas, dentre elas a pata-de-vaca. 
A regulação do metabolismo de carboidratos e liberação insulínica, segundo Figueiredo LB e Paiva PMH (2020), são ações imediatas obtidas pelo uso contínuo da pata-de-vaca, tal mecanismo regulatório é comprovado cientificamente, além de possuir ação inerente ao reparo e aumento do desempenho das células pancreáticas, atuando na captação eficaz da glicose. A atividade hipoglicemiante desta espécie vegetal consiste na liberação de insulina para recaptar moléculas de glicose e bloquear a absorção intestinal desta molécula, consequentemente, apresenta redução da glicemia e de mecanismos insulinodependentes, apresentando efeitos benéficos quanto os tratamentos farmacológicos, no entanto, com maior margem de segurança e poucos eventos adversos.

Segundo Silva HGN, et al. (2018), o uso de plantas medicinais no tratamento do Diabetes mellitus merece atenção especial dos profissionais de saúde envolvidos na linha de cuidado destes pacientes, de modo a oportunizar esses insumos para tratamento coadjuvante de patologias crônicas. Reforçando esta ideia, o exposto por Machado EO e Chaves ACTA (2018), em sua revisão bibliográfica, todos os artigos pesquisados fizeram referência a $B$. forficata no tratamento de diabetes, o que contribui para reflexões acerca da relevância desses estudos, fomento por mais pesquisas com plantas medicinais tão disponíveis na flora do território brasileiro, auxiliar na produção de medicamentos, além de dar sequência ao repasse de informações da medicina popular, uso empírico e saberes populares sobre as plantas que fazem parte da cultura amazônica e brasileira e merece destaque para registro e seguimento por várias gerações.

Com ação comprovada e uso cada vez mais frequente, o acompanhamento profissional é essencial, principalmente por médicos e farmacêuticos, oportunizando o uso racional destas plantas. Um alerta foi emitido nos estudos de Rodrigues JC, et al. (2019), em uma análise macroscópica e microscópica de amostras de folhas secas de $B$. forficata disponíveis para compra em casas de produtos naturais. O estudo demonstrou inúmeros produtos que continham rótulo da pata-de-vaca, no entanto, se tratava de outro espécime vegetal. Para evitar riscos à saúde, faz-se necessário sempre obter acompanhamento do médico e farmacêutico, auxiliando o tratamento seguro e efetivo.

\section{Estudos comprobatórios}

\section{Estudos in vitro}

Segundo Farias EL, et al. (2018), a pata-de-vaca é amplamente usada como hipoglicemiante, com efeitos renais. A planta medicinal destaca-se pela ação analgésica, depurativa, diurética, laxante, vermífuga, calmante e é benéfica para problemas renais e de grande importância para os diabéticos. Quanto aos testes realizados pelos autores, evidenciou-se que a $B$. forficata possui grande atividade antibacteriana frente a cepas de bactérias hospitalares multirresistentes mais frequentes no Brasil, e diante do risco iminente de resistência bacteriana, a planta medicinal pode representar um avanço e estratégia para combate da ação bacteriana.

Outros estudos de ordem experimental, como o proposto por Tonelli CA, et al. (2021), comprovaram que $300 \mathrm{mg}$ de extrato padronizado de folhas de $B$. forficata, administrados em cápsula, a dois grupos, placebo e $B$. forficata, no grupo que recebeu o extrato, as taxas de hemoglobina glicada e glicose plasmática foram significativamente menores no grupo $B$. forficata do que no grupo placebo. Este estudo sugeriu que o extrato padronizado da planta medicinal poderia ser utilizado como tratamento concomitante aos antidiabéticos orais no diabetes tipo 2.

A B. forficata apresentou homogeneidade como detentora de ação hipoglicemiante. O potencial terapêutico das plantas medicinais, segundo Cardoso RO, et al. (2019) é proveniente da reparação pancreática realizada pelos espécimes vegetais, resultando num aumento da liberação de insulina, além disso, a imensa quantidade de flavonóides constituintes deste espécime vegetal, principalmente a kaempferitrina realizam ação hipoglicemiante. A Bauhinia forficata apresentou ação na redução dos níveis de glicose em diferentes formas farmacêuticas, comprimidos, granulados, infusões e extratos aquosos, hexânicos e etanólicos, demonstrando flexibilidade e grandes oportunidades na criação de medicamentos. Outro mecanismo de ação decorre do bloqueio da absorção da glicose no lúmen intestinal (CARDOSO RO, et al., 2019).

Machado EO e Chaves ACTA, (2018) descrevem em suas revisões, achados imprescindíveis ao entendimento do uso popular da pata-de-vaca para o tratamento de diabetes. Segundo estes autores, extratos 
contendo folhas da planta acrescidos a hexano, água e etanol, resultaram na redução dos índices de glicose, triglicerídeos e colesterol total. Isto deve-se possivelmente a kaempferitrina, flavonóide responsável por sua ação hipoglicemiante. $O$ amplo referencial de pesquisas científicas serve como aporte para subsidiar a inclusão da $B$. forficata no âmbito da Atenção Primária à Saúde e fomenta pesquisas experimentais de produção de medicamentos, obtidos através da planta, para tratamento de diabetes mellitus.

Os estudos de Franco RR, et al. (2020), avaliaram a atividade de B. forficata em ensaios enzimáticos, constatando o potencial inibitório contra $\alpha$-amilase, $\alpha$-glucosidase e lipase, ação antioxidante e antiglicemiante. Nos ensaios, avaliaram efeitos citotóxicos da planta em macrófagos e eritrócitos de roedores, em que nenhuma fração apresentou efeitos citotóxicos e hemolíticos, justificando que $B$. forficata é uma planta segura no tratamento de diabetes mellitus. Por apresentar efetividade e segurança, esta planta está inclusa na Relação Nacional de Plantas Medicinais de Interesse ao SUS (RENISUS), apresentando grande potencial para gerar produtos de interesse ao Sistema único de Saúde (SUS).

\section{Estudos in vivo}

Cechinel-Zanchett CC, et al. (2019), demonstrando em uma pesquisa experimental com ratos que frações de compostos fenólicos contidos na espécie B. forficata, conhecida popularmente como pata-de-vaca, apresentou segurança, pouca interação com fármacos difundidos para o tratamento de diabetes, vasodilatação aórtica, redução dos níveis de glicose no sangue, redução do estresse oxidativo e efeito protetor cardiovascular, o que contribui para o reforçamento de que a pata-de-vaca pode ser usada para o tratamento de diabetes, além de ser auxiliar no tratamento de hipertensão arterial e desordens cardiovasculares.

\section{Estudos clínicos}

Na última década, diversos estudos com enfoque na descoberta de propriedades terapêuticas em plantas medicinais revelam o profundo interesse em explorar opções menos onerosas e produção de medicamentos efetivos, de baixo custo, ampla disponibilidade a população, redução dos efeitos colaterais e capacidade de controle de doenças crônicas. O estudo conduzido por Pinafo MS, et al. (2019), investigou o potencial terapêutico da $B$. forficata, evidenciando redução dos níveis de glicose induzidos pelo Bisphenol A (BPA), além de reduzir a elevação em triacilglicerol e frações VLDL (Very low-density lipoprotein) e LDL (Low Density Lipoproteins) do colesterol. Devido ao alto teor de flavonóides, a $B$. forficata diminuiu o estresse oxidativo no fígado, apresentou propriedades hipoglicêmicas e antioxidantes capazes de minimizar os efeitos do Bisphenol A (BPA).

Os estudos conduzem a afirmação de ação hipoglicemiante da $B$. forficata. Em uma pesquisa realizada por Silva HGN, et al. (2018), levando em consideração aspectos socioculturais e clínicos que podem influenciar no uso de plantas medicinais por idosos com Diabetes Mellitus, detectou que 71 idosos cadastrados no programa HIPERDIA - de controle a hipertensão arterial, 94,3\% dos participantes da pesquisa tinham diabetes associada à Hipertensão Arterial, em 66,19\% dos idosos relataram usar plantas medicinais para o tratamento da diabetes, dentre as plantas, a infusão das folhas da $B$. forficata foi a mais utilizada, em $38,29 \%$ dos idosos.

Souza ID, et al. (2020) avaliou em um relato de caso um paciente portador de diabetes mellitus há mais de 30 anos, no qual foi inserido o tratamento com extrato de $B$. forficata concomitante ao tratamento farmacológico. $O$ tratamento com $B$. forficata apresentou atividade anti-inflamatória, redução da hemoglobina glicada, glicose em jejum, pré e pós-prandial. O paciente em uso não deixou de tomar suas medicações hipoglicemiantes orais, no entanto, inseriu um tratamento paralelo, o que auxiliou no tratamento da doença crônica, possibilitou saúde e qualidade de vida.

Oliveira DC, et al. (2020) fez avaliação de 188 pacientes portadores de diabetes mellitus tipo 2, que utilizaram por um período de tempo a infusão proveniente de $B$. forficata, os resultados foram similares aos descritos por outros estudos supracitados, ampla ação hipoglicemiante, redução do estresse oxidativo, níveis de colesterol, triglicerídeos e risco de problemas cardiovasculares, reforçando a planta como agente promissor no desenvolvimento de fármacos utilizados no tratamento de diabetes mellitus. 
Os achados de Pontes MAN, et al. (2017) e Prado IO, et al. (2020) finalizam este estudo de revisão, com a difusão de efetividade e segurança da $B$. forficata no tratamento de diabetes mellitus, principalmente os efeitos sobre os portadores de diabetes tipo 2 e gestacional. Segundo Pontes MAN, et al. (2017), diante do conhecimento da ação terapêutica da pata-de-vaca, descreve que os estudos clínicos com a planta tiveram início na década de 1920, comprovando ação hipoglicemiante. Em seu estudo de revisão, entre os anos de 2007 a 2017, evidenciou-se efeitos hipoglicemiantes do extrato da B. forficata em diferentes formas farmacêuticas, desde pós a granulados e enaltece a planta como potencial promissor para o desenvolvimento de medicamentos em diferentes formas farmacêuticas, capazes de conduzir ao uso seguro, racional, trazer credibilidade e aumentar as chances de utilização, principalmente por idosos portadores de diabetes mellitus, público, por vezes, que apresenta alta faixa de não-adesão ao tratamento, por ordens diversas.

\section{CONSIDERAÇÕES FINAIS}

De acordo com os artigos pesquisados neste estudo, a pata-de-vaca apresenta ampla ação hipoglicemiante, devido aos flavonóides constituintes. A Bauhinia forficata apresentou ação na redução dos níveis de glicose em diferentes formas farmacêuticas, comprimidos, granulados, infusões e extratos aquosos, hexânicos e etanólicos, demonstrando flexibilidade e grandes oportunidades na criação de medicamentos. Este estudo contribui para substanciar embasamento científico para estudos futuros, bem como, para a afirmação acerca da ação hipoglicemiante da Bauhinia forficata, principalmente no contexto da região amazônica, a qual insere-se este estudo, onde a utilização da pata-de-vaca no tratamento de diabetes é antiga e se traduz em um saber empírico da medicina popular, sapiência utilizada e repassada por muitas gerações, mas efetiva e condutora de qualidade de vida e redução das complicações associadas ao diabetes mellitus.

\section{REFERÊNCIAS}

1. BRÍGIDO HPC, et al. (2021). Evaluation of acute and subacute toxicity of ethanolic extract and fraction of alkaloids from bark of Aspidosperma nitidum in mice. Scientific Reports, 2021; 11(1), 1-14.

2. BRITO VP, et al. A fitoterapia como uma alternativa terapêutica complementar para pacientes com Diabetes Mellitus no Brasil: uma revisão sistemática. Saúde e meio ambiente: revista interdisciplinar, 2020; 9: 189-204.

3. CARDOSO RO, et al. Efeito hipoglicemiante da canela (Cinnamomum sp.) e pata-de-vaca (Bauhinia $s p$.): Revisão Bibliográfica. Arquivos do MUDI, 2019; 23(3): 399-412.

4. CARVALHO AC, et al. Plantas medicinais utilizadas no tratamento do Diabetes Mellitus: Uma revisão. Brazilian Journal of Health Review, 2021; 4(3): 12873-12894.

5. CECHINEL-ZANCHETT CC, et al. Bauhinia forficata link, a Brazilian medicinal plant traditionally used to treat cardiovascular disorders, exerts endothelium-dependent and independent vasorelaxation in thoracic aorta of normotensive and hypertensive rats. Journal of ethnopharmacology, 2019; 243: 112-118.

6. FARIAS FL, et al. Avaliação da atividade antibacteriana de extrato etanólico da Bauhinia forficata L. Diversitas Journal, 2018; 3(2): 402-411.

7. FIGUEIREDO LB, PAIVA PMH. Levantamento sobre a utilização de plantas medicinais por universitários e colaboradores do centro Universitário do Sul de Minas -Varginha MG. Brazilian Journal of Development, 2020; 6(12): 101718-101735.

8. FONSECA KP e RACHED CDA. Complicações do diabetes mellitus. International Journal of Health Management Review, 2019; 5(1): 1-13.

9. FRANCO RR, et al. Antidiabetic potential of Bauhinia forficata Link leaves: a non-cytotoxic source of lipase and glycoside hydrolases inhibitors and molecules with antioxidant and antiglycation properties. Biomedicine e Pharmacotherapy, 2020; 123: 1097-1098.

10. GUIMARÃES BM, et al. Práticas terapêuticas com plantas medicinais para o tratamento do Diabetes Mellitus. Research, Society and Development, 2021; 10(10): e474101018874.

11. JUNG EP, et al. Recuperação termicamente assistida de compostos antioxidantes de folhas de Bauhinia forficata: efeito das condições operacionais. Jornal de pesquisa aplicada em plantas medicinais e aromáticas, 2021;22: 100303.

12. 11.MACHADO EO, CHAVES ACTA. Plantas utilizadas no tratamento do diabetes tipo II: uma revisão de literatura. Revista Textura, 2018; 11(20): 47-55.

13. MARIÁNGEL PC, et al. Efeitos do chá Bauhinia forficata Link no perfil lipídico em pacientes diabéticos. Jornal de alimentos medicinais, 2019; 22 (3), 321-323. 
14. NASCIMENTO EMM, et al. Efeito da suplementação do Momordica charantia L. em pacientes com Diabetes mellitus: Uma revisão sistemática. Research, Society and Development, 2021; 10 (6): e2110615143.

15. OLIVEIRA DC, et al. Use of Bauhinia forficata Link infusion in the treatment of diabetes mellitus. Research, Society and Development, 2020; 9(10): e3159108574.

16. PINAFO MS, et al. Effects of Bauhinia forficata on glycaemia, lipid profile, hepatic glycogen content and oxidative stress in rats exposed to Bisphenol A. Toxicology reports, 2019; 6: 244-252.

17. PONTES MAN, et al. Bauhinia forficata L. e sua a ação hipoglicemiante. Archives of Health Investigation, 2017; 6(11): 509-512.

18. PRADO IO, et al. Efeitos metabólicos da infusão das folhas de Bauhinia forficata em Drosophila melanogaster alimentadas com uma dieta rica em sacarose. Research, Society and Development, 2020; 9(3): 16.

19. RODRIGUES JC, et al. Avaliações da qualidade da pata-de-vaca comercializada no Brasil. Revista Agrogeoambiental, $2019 ; 11$ (3).

20. SANTOS AL, et al. Adesão ao tratamento de diabetes Mellitus e relação com assistência na atenção primária. Revista Mineira de Enfermagem, 2020; 24: 1-10.

21. SILVA HGN, et al. Retrato sociocultural: o uso de plantas medicinais por pacientes idosos com diabetes mellitus tipo 2. Revista Interdisciplinar, 2018; 11(4): 21-29.

22. SILVA AD, et al. Estado nutricional, fatores de risco e comorbidades em adultos portadores de diabetes mellitus tipo 2. HU Revista, 2020; 46: 1-9.

23. SOCIEDADE BRASILEIRA DE DIABETES. Diretrizes da Sociedade Brasileira de Diabetes 2019-2020. Disponível em: http://www.saude.ba.gov.br/wp-content/uploads/2020/02/Diretrizes-Sociedade-Brasileira-de-Diabetes-2019-2020.pdf. Acesso: 31 de jan.

24. SOUZA ID, et al. A Case Report of Diabetes Mellitus Type 2 with Adjuvant Use of Bauhinia forficata in Pharmacological Treatment. European Journal of Medicinal Plants, 2020; 31(15): 49-56.

25. TONELLI CA, et al. Clinical efficacy of capsules containing standardized extract of Bauhinia forficata Link (pata-devaca) as adjuvant treatment in type 2 diabetes patients: A randomized, double blind clinical trial. Journal of Ethnopharmacology, 2022; 282: 114616.

26. XAVIER AT, NUNES JS. Tratamento de diabetes mellitus com plantas medicinais. Revista Científica Da Faculdade De Educação E Meio Ambiente, 2018; 9(edesp): 603-609. 